\title{
Research progress of functional lactic acid bacteria
}

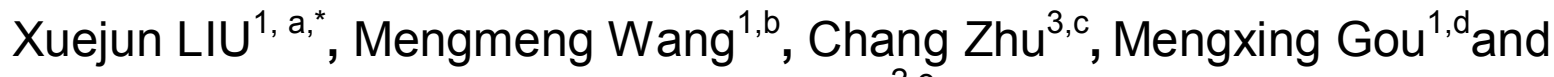 \\ Xiaohui Yan ${ }^{2, e}$ \\ ${ }^{1}$ College of Food Science and Engineering, Jilin Agricultural \\ University, Changchun, Jilin,130118,China \\ 2 Jilin Business and Technology College, Changchun, Jilin, 130507, China \\ ${ }^{3}$ Jilin Engineering Vocational College, Food Engineering Branch,Siping, Jilin,136000, China

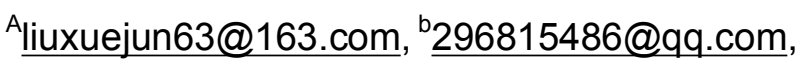 \\ c14170567@qq.com, ${ }^{\mathrm{d}}$ 122545310@qq.com, ${ }^{\mathrm{e}}$ 1079128608@qq.com
}

\begin{abstract}
Keywords: Lactic acid bacteria, function, antioxidation, decrease cholesterol
Abstract. Lactic acid bacteria are closely related to health, in recent years, experts and scholars at home and abroad have been continuously exploring the function of lactic acid bacteria. This article introduced antioxidant, decrease cholesterol, reduce blood press, immune function, produce bacteriocin, and compose vitamins functions of lactic acid bacteria. To provide reference for further development and utilization of functional lactic acid bacteria
\end{abstract}

\section{Introduction.}

Lactic acid bacteria are gram positive bacteria, non spore, anaerobic, the use of carbohydrates to produce lactic acid bacteria ${ }^{[1]}$, can be divided into 18 genera, a total of more than 200 kinds. At present, it has been confirmed by domestic and foreign biologists that there is a very close relationship between lactic acid bacteria and health.

First lactic acid bacteria separated from the milk, often found in some food and fermentation products in nature, in nature, there are many kinds and wide distribution. Most of them can can ferment lactate are bacteria, some are coccus, some are bacillus, generally do not exercise. They take monosaccharide and disaccharide as substrate, The mainly metabolites are lactic acid, some lactic acid bacteria belong to resistance of anaerobic microorganisms, although they are not aerobic capacity, they can be a typical lactic acid fermentation under anaerobic conditions ${ }^{[2]}$.

The main function of lactic acid bacteria are antioxidant; improve human intestinal function, recovery the balance of human intestinal flora, especially by producing bacteriocin, inhibition of intestinal pathogenic bacteria specific growth and toxin production, balanced intestinal flora; prevention of cardiovascular diseases, including cholesterol lowering effect and reduce the possibility and effect of anti-hypertension, decrease the possibility of occurrence of hypercholesterolemia, atherosclerosis, coronary heart disease and other high-risk disease; enhance human immunity; inhibit the growth of spoilage bacteria; anti tumor; nervous system anti-aging function, recently found that probiotics can regulate the neurotransmitter to prevent Alzheimer's disease and other pathological aging; synthetic vitamins; preservation food; improve food flavor and so on ${ }^{[3]}$.

\section{Antioxidant}

Oxidation is necessary for the most of living organisms, but it will also destroy the biological molecules, causing great harm to life. The antioxidant activity of lactic acid bacteria has been verified by in vivo and in vitro experiments. Lactic acid bacteria will become a new type of antioxidant. In recent years, the research on the antioxidant activity of lactic acid bacteria has been carrying out at home and abroad. Ebel et al. ${ }^{[4]}$ studied on the antioxidant activity of lactic acid bacteria isolated ME-3 obtained from healthy infants in the gut, the results showed that the lactic acid bacteria containing glutathione and manganese superoxide dismutase (Mn-SOD), which can eliminate the hydroxyl radicals, explained the lactic acid bacteria is an effective cellular antioxidant. 
Murtaza et al. ${ }^{[5]}$ study showed that lactic acid bacteria fermented soybean milk can effectively eliminate free radicals.Ming Chen et al. ${ }^{[6]}$ studied that 3 strains of lactic acid bacteria isolated from the dairy cows in the Qinghai Tibet Plateau have high antioxidant activity. Yaoqing Li et al. ${ }^{[7]}$ studied that antioxidant activity of different strains of mouse colitis model induced by dextran sulfate sodium (DSS). The results showed that lactic acid bacteria Fn008 and Fn037 could significantly inhibit the content of ROS in colon and increase the total antioxidant capacity of colon. Bae et al. $^{[8]}$ reported in the study, BJ-20 strains which isolated from the fermentation plants identified as Lactobacillus brevis, the strains in the fermentation process of seaweed, exhibited strong antioxidant activity, and the antioxidant activity is better than natural antioxidants butyl hydroxy anisole (BHA).

\section{Decrease cholesterol}

Research shows that high serum cholesterol level, easily lead to vascular atherosclerosis and coronary heart disease, stroke, hypertension and other cardiovascular and, seriously threat to human health. Therefore, decreasing the cholesterol levels of serum and food is one of the hot spots of scientific research. Lactic acid bacteria and their related products have the ability to reduce cholesterol in the medium and serum, which has been confirmed by a large number of in vivo and in vitro experiments. Huang $\mathrm{Y}$ et al. ${ }^{[9]}$ studied that probiotic lactobacillus acidophilus ATCC4356 regulated Niemann-Pick C1-like 1 NPC1L1, when weak expression, significantly reduce the intestinal absorption of cholesterol. Jeun $\mathrm{J}$ et al. ${ }^{[10]}$ used double embedding lactobacillus plantarum KCTC3928 in high fat diet intake mice, found that the bacteria can reduce the serum and liver cholesterol by accelerating the synthesis of bile acid and increasing the excretion of fecal bile acid, thereby protecting the liver function. Hao Dong et al. ${ }^{[1]}$ taken sauerkraut juice as experimental materials to screen cholesterol reducing lactic acid bacteria, The mice were fed with the mixture of this strain and schisandra chinensis decoction, the results showed that the mixture of the lactic acid bacteria and schisandra fruit decoction has the significantly effect of lowering cholesterol. Xiuhong Lv et al. ${ }^{[12]}$ screened two strains of lactobacillus plantarum with high cholesterol degradation rate from traditional food, and the cholesterol degradation rate reached to $49.11 \%$ and $50.03 \%$, which laid a good foundation for further development and application.

\section{Reduce blood pressure}

Hypertension is one of the main diseases endangering human health, is the most common cardiovascular disease, its incidence rate is high, often accompanied by arteriosclerosis, coronary heart disease and kidney failure, caused a great threat to people's health. At home and abroad, it has been found that a variety of lactic acid bacteria have the function of lowering blood pressure. Yu et al. ${ }^{[13]}$ studied that on the effect of lactic acid bacteria isolated from kimchi on anti-hypertensive , inhibit the activity of angiotensin converting enzyme (ACE), and dealed spontaneously hypertensive rats with lactobacillus. The results showed that the extract of strain LG42 had significant inhibitory effect on hypertension in mice. Osuntoki et al. ${ }^{[14]}$ fed male rats and blank control group with the milk which fermented by lactobacillus acidophilus separated by the indigenous high salt fermented food and high salt food ,to comparative anti hypertensive effect of lactobacillus. The results showed that the isolated lactobacillus has the function of anti-hypertension. Gobbeiti et al. ${ }^{[15]}$ found that small peptides were isolated from fermented milk of lactobacillus bulgaricus (L.bulgaricus SS1) and lactobacillus (L.lactis cremorisFT4)

\section{Immune function}

Lactic acid bacteria as the main dominant bacteria in the intestines of healthy people and animals play an important role in the regulation of intestinal immune function and intestinal mucosal barrier. The results of Li Xueping et al. ${ }^{[16]}$ showed that lactic acid bacteria can improve the production performance, intestinal health and immune function of broilers. The results of Chuang et al. ${ }^{[17]}$ 
showed that inactivation of lactic acid bacteria can stimulate cell proliferation and interleukin IL-10, IL-12 P70, interferon IFN- gamma production, and increase the secretion of T cell TH. Beatriz et al. ${ }^{[18]}$ showed that the expression of Osp $\mathrm{A}$ in lactobacillus plantarum promoted the secretion of cytokine IL- 8 in intestinal epithelial cells, and the intestinal immune tolerance was broken by Th1/Th2 cell-mediated immunity. Li Li et al. ${ }^{[19]}$ studied that take orally lactic acid bacteria can inhibit dust mite induced allergic airway inflammation, is mainly regulated the proliferation of $\mathrm{T}$ cell subsets of mouse spleen cells induced by lactic acid bacteria, through the release of IL-10 to down regulation cytokine levels of Th1/Th2. Shaomin Feng et al. ${ }^{[20]}$ studied the specific lactic acid bacteria (Lb-f) dose dependently increased the titer of mouse anti sheep erythrocyte agglutinin antibody, and enhanced the humoral immune function.

\section{Produce bacteriocin}

Lactobacillus bacteriocin is a kind of bacteriocins produced by lactic acid bacteria metabolism has antibacterial effects of natural proteins or polypeptides, the bacteriocins as antibacterial substances mixed with food systems has been studied extensively, With a large amount of data show that the bacteriocin has the power of control of spoilage microorganisms. The potential application of bacteriocins in food preservation and health care has aroused great interest of scholars at home and abroad. Arokiyamary $\mathrm{A}^{[21]}$ isolated a bacteriostatic from milk and cheese has effect on Staphylococcus aureus, Bacillus cereus, Bacillus typhi and other strains, identified as lactobacillus. After excluding the effects of organic acids and hydrogen peroxide, determination of the antibacterial substance was a kind of bacteriocins produced by lactic acid bacteria. Tejpal Dhewa ${ }^{[22]}$ purified and separated bacteriocin from lactic acid bacteria and lactic acid bacteria, and prove that bacteriocins has a bactericidal effect on Staphylococcus aureus in milk and other food pathogenic bacteria. Peng Gao et al. ${ }^{[23]}$ isolated a strain from Heilongjiang traditional homemade sauerkraut named lactic acid bacteria HLJ-174 can produce antibacterial substances, the active substances have strong antibacterial activity against Escherichia coli and Bacillus cereus.

\section{Compose vitamins}

Vitamin is a kind of small molecule organic compound, which is necessary to maintain normal life, long-term lack of any kind of vitamin will lead to the corresponding disease. It is reported that lactic acid bacteria can synthesize $\mathrm{B}_{12}$ and folic acid during metabolism, the vitamin fermented by lactic acid bacteria can reduce the side effects of synthetic vitamins, with good safety. Crittenden RD et al. ${ }^{[24]}$ used microbiological method to determine the ability of 32 strains of fermentation bacteria and probiotics compose folic acid during the fermentation of skim milk. It was found that the production of folic acid was highest in Streptococcus thermophilus. Lactobacillus is usually used prophase time to produce own folic acid in skim milk. After fermentation of skim milk with bifidobacterium and streptococcus thermophilus, folic acid concentration increased by 6 times. Taranto et al. ${ }^{[25]}$ isolated strains from sourdough can produce cobalamin $\left(\mathrm{B}_{12}\right)$ named lactobacillus reuteri CRL1098. Utilizing microbiological method, the concentration of vitamin B12 in cell extracts was measured to be about $0.5 \mathrm{mg} / \mathrm{L}$, using the inverse of HPLC of lactobacillus reuteri produced in the form of isolated cobalamin to cyanide. This product is in accordance with the standard product of UV - visible spectra of cyanocobalamin. Santos et al. ${ }^{[26]}$ studied the effects of culture conditions on lactobacillus reuteri JCM1112 production of vitamin $\mathrm{B}_{12}$, found that the use of single amino acid deletion method (lack of isoleucine) can produce a significant increase in $\mathrm{B}_{12}$ level. This method provides a new way to improve the yield of vitamin $\mathrm{B}_{12}$.

In this article, several typical functional characteristics of lactic acid bacteria are discussed. The function of lactic acid bacteria will play an important role in human health, but more works neede to confirm the mechanism of lactic acid bacteria on human body at the molecular level. The function of lactic acid bacteria is still being explored, and it is necessary to further study the mechanism of lactic acid bacteria. 


\section{References}

[1] Anna H.: Eoless,Budapest. Sci. (2009), p.70-83

[2] Suresh K. A., Jaishree P.: Current Scienc. Sci. . Vol. 80 (2001), p.1310-1312

[3] Yin SL, Du J, Xu C.: Food Sci Technol. Sci. Vol. 37 (2012), p.25-29

[4] Ebel M.E., Awe O., Kaplan M. H.: The Journal of Immunology. Sci. Vol. 194 (2015), p.5781-5788

[5] Murtaza N., Baboota R. K., Jagtap S.: British Journal of Nutrition. Sci. Vol. 112 (2014), p.1447-1458

[6] Ming Chen, Wencan Ke, Anan Bao: Food industry technology.In Chinese. Vol. 37 (2016), p.201-205

[7] Yaoqing Li, Jin Sun, Guowei Yao: Chinese Journal of Microecology. In Chinese. (2016), p.97-100

[8] Bae J. L., Jin S. K., Young M. K.: Food Chemistry. Sci. (2010), p.271-276

[9] Huang Y., Zheng YC.: British J Nutr. Sci. Vol. 103 (2010), p.473-478

[10]Jeun J.: Nutrition. Sci. (2010), p.321-330

[11]Hao Dong, Hongli Wang, Xin Wang: Journal of Changchun University. In Chinese. Vol. 26 (2016), p.53-58

[12]Lv Xiuhong, Kaifei Chen,Qi Zhu,: Journal of Chinese Institute of Food Science and Technology. In Chinese. Vol. 16 (2016), p.198-204

[13] Yu MH, Im HG, Im NK: Korean J Food. Sci. Vol. 41 (2009), p.428-434

[14] Osuntoki AA, Omonigbehin EA, Gbenebitse SO: Nigerian Food J. Sci. Vol. 25 (2007), p.184-189

[15]Gobbeiti M., Ferranti P., Smacchi E.: Appl Enviorn Micorbiol. Sci. Vol. 66 (2000), p.3898-3904

[16]Li XP, Ji C., Liu AJ: Chin Feed Add. Sci. (2011), p.29-32

[17]Chuang L.,Wu KG, Pai C.: J Agric Food Chem. Sci. (2007), p.11080-11086

[18]Beatriz del R, Jos FS, Maria GS: Plos One. Sci. Vol. 5 (2010), p.1-10

[19]Li Li, Lin Cai, Baodan Yu: Journal of Immunology. In Chinese. Vol. 27 (2011), p.193-198

[20] Shaomin Feng, Hong Xia,: Clinical Medicine. In Chinese. Vol. 30 (2010), p.102-103

[21] Arokiyamary A.,Sivakumar P K.: Journal of Pharmtech Research. Sci. Vol. 4 (2012), p.315-320

[22]Tejpal D.: International Conference on Nutrition and Food Sciences. Sci. Vol. 39 (2012), p.35-41

[23]Peng Gao, Jinzhi Han, Zhaoxin: Food Science In Chinese. Vol. 37 (2016), p.160-166

[24]Crittenden RG: Int J Food Microbiol. Sci. (2003), p.217-222

[25] Taranto MP: J Bacteriol. Sci. (2003), p.5643-5647

[26] Santos F.: Appl Environ Microbiol. Sci. (2009), p.3930-3936 